\title{
Subsets of Inflammatory Cytokine Gene Polymorphisms are Associated with Risk of Carcinogenic Liver Fluke Opisthorchis viverrini-Associated Advanced Periductal Fibrosis and Cholangiocarcinoma
}

\author{
Arpa Surapaitoon ${ }^{1,2}$, Sutas Suttiprapa ${ }^{2,3}$, Eimorn Mairiang ${ }^{4}$, Narong Khuntikeo ${ }^{5}$, Chawalit Pairojkul ${ }^{3}$, \\ Jeffrey Bethony ${ }^{6}$, Paul J. Brindley ${ }^{6}$, Banchob Sripa ${ }^{2,3, *}$ \\ ${ }^{1}$ Biomedical Sciences, Graduate School, Khon Kaen University, Khon Kaen, Thailand; ${ }^{2}$ WHO Collaborating Centre for Research and Control of \\ Opisthorchiasis (Southeast Asian Liver Fluke Disease), Tropical Disease Research Center; ${ }^{3}$ Department of Pathology; ${ }^{4}$ Department of Radiology, \\ Faculty of Medicine, Khon Kaen University, Khon Kaen, Thailand; ' Liver Fluke and Cholangiocarcinoma Research Center, and Department of \\ Surgery, Faculty of Medicine, Khon Kaen University, Khon Kaen 40002, Thailand; ${ }^{6}$ Department of Microbiology, Immunology and Tropical Medicine, \\ and Research Center for Neglected Tropical Diseases of Poverty, School of Medicine \& Health Sciences, The George Washington University, \\ Washington DC, 20037, USA
}

\begin{abstract}
Opisthorchis viverrini infection induces chronic inflammation, and a minor proportion of infected individuals develop advanced periductal fibrosis (APF) and cholangiocarcinoma (CCA). Inflammatory cytokines and/or their gene polymorphisms may link to these biliary pathologies. We therefore investigated associations among cytokine gene polymorphisms and cytokine production in 510 Thai cases infected with O. viverrini who presented with APF+ or APF-, as established by abdominal ultrasonography as well as in patients diagnosed with CCA. Levels of pro-inflammatory and anti-inflammatory cytokines were determined in culture supernatants after stimulation of peripheral blood mononuclear cells (PBMCs) with O. viverrini excretory-secretory (ES) products. Pro-inflammatory cytokines, IL-1 $\beta$, IL-6, IFN- $\gamma$, LT-a, and TNF-a were significantly increased in CCA patients compared with non-CCA (APF- and APF+) cases. Polymorphisms in genes encoding IL-1 $\beta-511 \mathrm{C} / \mathrm{T}$, IL-6 -174G/C, IFN- $\gamma+874 \mathrm{~T} / \mathrm{A}$, LT- $\alpha+252 \mathrm{~A} / \mathrm{G}$, and TNF- $\alpha-308 \mathrm{G} / \mathrm{A}$ were then investigated by using PCR-RFLP or allele specific-PCR (AS-PCR) analyses. In the CCA cases, LT- $a+252 A / G$ and TNF- $a-308 G / A$ heterozygous and homozygous variants showed significantly higher levels of these cytokines than the wild type. By contrast, levels of cytokines in wild type of IFN- $\gamma+874$ T/A were significantly higher than the variants in CCA cases. IFN- $\gamma+874 T / A$ polymorphisms were associated with advanced periductal fibrosis, whereas IL-6 -174G/C polymorphisms were associated with CCA. To our knowledge, these findings provide the first demonstration that $O$. viverrini infected individuals carrying several specific cytokine gene polymorphisms are susceptible to develop fibrosis and CCA.
\end{abstract}

Key words: Opisthorchis viverrini, hepatobiliary disease, advanced periductal fibrosis, cholangiocarcinoma, cytokine gene polymorphisms

\section{INTRODUCTION}

Chronic infection with the carcinogenic liver fluke Opisthorchis viverrini results in continuous irritation, chronic inflammation, restlessness tissue damage, and repair of the bile tract, including the second order bile ducts [1]. Consequently, infection with $O$. viverrini is strongly associated with chronic hepatobiliary disease, including cholangitis, cholecystitis, obstruc-

\footnotetext{
- Received 18 April 2017, revised 8 May 2017, accepted 11 May 2017.

*Corresponding author (banchob@kku.ac.th)

(C) 2017, Korean Society for Parasitology and Tropical Medicine

This is an Open Access article distributed under the terms of the Creative Commons Attribution Non-Commercial License (http://creativecommons.org/licenses/by-nc/4.0) which permits unrestricted non-commercial use, distribution, and reproduction in any medium, provided the original work is properly cited.
}

tive jaundice, advanced periductal fibrosis, and cholangiocarcinoma (CCA) [1,2]. Ultrasonographic assessment of hepatobiliary abnormalities of otherwise asymptomatic persons stool positive for infection with O. viverrini revealed that about 25\% of these cases developed advanced periductal fibrosis, and CCA was suspected in up to $0.5 \%$ of them based on abdominal ultrasound examinations $[3,4]$. The mechanism underlying the different outcomes of $O$. viverrini infection is unknown. Our extensive research experience with opisthorchiasis led us the suspect that host inflammatory responses to the liver fluke might play pivotal roles in the outcome [1,2]. Inflammation mediated by cytokines has been described in infectious diseases and malignancies $[5,6]$. Specifically, the liver fluke infection induces marked inflammation characterized by infiltration by 
neutrophils and mononuclear cells into the vicinity of the infected bile ducts. Proteins and other metabolites released by the O. viverrini worms activate Toll-like receptor 4 and induce expression of the potent chemo-attractant IL-8 and pro-inflammatory IL-6 cytokines [7].

Gene polymorphisms affect the cytokine expression and influence inflammatory processes in response to infectious diseases and cancer [8]. In the context of liver fluke infection, hepatobiliary disease and associated bile duct cancer, we have reported marked elevation in expression of IL-6 in response to O. viverrini excretory-secretory (ES) antigens during chronic opisthorchiasis, particularly where biliary periductal fibrosis is marked [9]. In addition, we observed significant increases in both pro-inflammatory and anti-inflammatory cytokines during cholangiocarcinoma associated with chronic liver fluke infection [10]. We now hypothesize that cytokine gene polymorphisms influence cytokine expression profiles, which in turn may result in diverse pathogenic sequelae during infection with $O$. viverrini. This study described below investigated associations among cytokine gene polymorphisms and cytokine production during chronic opisthorchiasis and associated biliary tract diseases, including CCA.

\section{MATERIALS AND METHODS}

\section{Study participants}

This study was approved by the Khon Kaen University Human Ethics Committee, reference nos. HE480528 and HE 581272. Written informed consents were obtained from all participants or patients. Four hundred participants positive for infection with O. viverrini, including 200 without APF- and 200 with $\mathrm{APF}+$, were recruited from sites endemic for O. viverrini infection in Khon Kaen or neighboring provinces in northeastern Thailand, as described [9]. This study also included 110 histologically proven cases of cholangiocarcinoma (CCA); these latter participants had undergone hepatectomy at Srinagarind Hospital, Faculty of Medicine, Khon Kaen University, Khon Kaen, Thailand.

\section{Opisthorchis viverrini-ES product preparation}

ES products of $\mathrm{O}$. viverrini $(\mathrm{O} v$-ES) were prepared as described $[11,12]$. Briefly, viable liver flukes recovered at necropsy from experimentally infected hamsters were cultured in RPMI1640 containing antibiotics and the protease inhibitors (0.1 $\mathrm{mM}$ phenylmethanesulfonyl fluoride (PMSF), $1 \mathrm{mM}$ leu- peptin and $0.1 \mathrm{mM} \mathrm{N}$-[N-(L-3-trans-carboxyoxiran-2-carbonyl)- L-leucine]-agmatine, E-64). The cultures were maintained in vitro at $37^{\circ} \mathrm{C}$ and supernatants containing the $\mathrm{O} v$-ES were collected twice daily for 7 days. The culture supernatants were subjected to centrifugation at 3,000 $\mathrm{g}$ for $10 \mathrm{~min}$ to pellet the eggs. Clarified supernatants were pooled, dialyzed against PBS, and concentrated by membrane ultrafiltration (PM 10, Amicon, Danvers, Masssacusetts, USA). Lipopolysaccharide (LPS) was removed using Triton-X114 extraction [13], followed by passage through Bio-Beads SM2 (Bio-Rad, Hercules, CA, USA) to remove Triton-X114. Finally, OvES was filtered through a 0.2 $\mu \mathrm{m}$ membrane and then aliquoted and stored at $-80^{\circ} \mathrm{C}$. The LPS concentration was determined using the Limulus amebocyte assay (less than $100 \mathrm{ng} / \mathrm{ml}$ failed to affect cell proliferation or cytokine production, unpublished) [14].

\section{Peripheral blood mononuclear cells (PBMCs) culture}

PBMC were isolated from heparinized blood by density gradient centrifugation using Ficoll-Paque (Sigma Diagnostic, St. Louis, Missouri, USA), counted on a hematocytometer and viability assessed using $0.5 \%$ Trypan blue dye. PBMC were cultured at $2 \times 10^{4}$ cells $/ \mathrm{ml}$ in RPMI-1640 supplemented with antibiotics and 5\% fetal bovine serum with or without $\mathrm{Ov}$-ES, 20 $\mu \mathrm{g} / \mathrm{ml}$. Phytohemaglutinin (PHA)-L (Difco Laboratories, Detroit, Michigan, USA) at $10 \mu \mathrm{g} / \mathrm{ml}$ was used as positive control of cytokine stimulation. The cells were cultured at $37^{\circ} \mathrm{C}$ in a humidified 5\% $\mathrm{CO}_{2}$ incubator for $48 \mathrm{hr}$. Supernatants were harvested and stored at $-80^{\circ} \mathrm{C}$ for cytokine level determination.

\section{Cytokine detection}

A 25-50 $\mu$ l of supernatant was used for cytokine assays. The secreted cytokines were quantified using the FlowCytomix bead-based multiplexing assays kit, human Th1/Th2 (IFN- $\gamma$, IL-1 $\beta$, IL-2, IL-4, IL-5, IL-6, IL-8, IL-10, IL-12p70, TNF- $\alpha$, and LT- $\alpha$ ) 11-plex kit (BMS810FF, BenderMedSystems, Vienna, Austria) and quantified using flow cytometry (FC500, BeckmanCoulter, Brea, California, USA). Cytokine levels were established by interpolation from standard curves established using human recombinant cytokines (BMS8400FF, BenderMedSystems), after which the findings were expressed as $\mathrm{pg} / \mathrm{ml}$.

\section{DNA extraction and genotyping}

Genomic DNA was extracted from PBMC from each participant using the Puregene DNA Isolation Kit (Gentra systems, Minneapolis, Minnesota, USA), quantified using the Nano- 
Table 1. Primer sequences, restriction enzymes, and size of digestion products in the PCR-RFLP and AS-PCR analyses

\begin{tabular}{|c|c|c|c|c|c|c|}
\hline Gene/SNP & rsn & wt/var & Region & Oligonucleotide primers & $\mathrm{RE}$ & Digestion products (bp) \\
\hline IL-1及/-511 & rs16944 & $\mathrm{C} / \mathrm{T}$ & Promoter & $\begin{array}{l}\text { F (5'- TGG CAT TGA TCT GGT TCA TC-3') } \\
\text { R (5'-GTT TAG GAA TCT TCC CAC TT-3') }\end{array}$ & Ava I & $\begin{array}{l}\text { wt: } 190,114 \\
\text { var: } 304\end{array}$ \\
\hline IL-6/-174 & rs1800795 & $\mathrm{G} / \mathrm{C}$ & Promoter & $\begin{array}{l}\text { F (5'-GGG CTG CGA TGG AGT CGA AG-3') } \\
\text { R (5'-TCC CTC ACA CAG GGC TCG-3') }\end{array}$ & Nla III & $\begin{array}{l}\text { wt: } 233,54 \\
\text { var: } 122.111,54\end{array}$ \\
\hline IFN- $\gamma /+874$ & rs2430561 & T/A & Intron 1 & $\begin{array}{l}\text { Common primer: 5'TCAACAAAGCTGATACTCCA-3', } \\
\text { T allele primer: 5'TTCTTACAACACAAAATCAAATCT-3', } \\
\text { A allele primer: 5'TCTTACAACACAAAATCAAATCA-3' }\end{array}$ & & \\
\hline $\mathrm{LT}-\mathrm{a} /+252$ & rs909253 & $A / G$ & intron & $\begin{array}{l}\text { F (5'-CCG TGC TTC GTG CTT TGG ACT A-3') } \\
\text { R (5'-AGA CGT TCA GGT GGT GTA AT-3') }\end{array}$ & Ncol & $\begin{array}{l}\text { wt: } 427 \\
\text { var: } 231,196\end{array}$ \\
\hline TNF-a/-308 & rs1800629 & $\mathrm{G} / \mathrm{A}$ & promoter & $\begin{array}{l}\text { F (5'-TCC TCC CTG CTC CGA TTA CG-3') } \\
\text { R (5'-AGG CAA TAG GTT TTG AGG GCC-3') }\end{array}$ & Ncol & $\begin{array}{l}\text { wt: } 87 \\
\text { var: } 107\end{array}$ \\
\hline
\end{tabular}

SNP, Single nucleotide polymorphism; rsn, reference SNP number; wt, wild type; var, variant; RE, restriction enzyme; bp, base pair

A

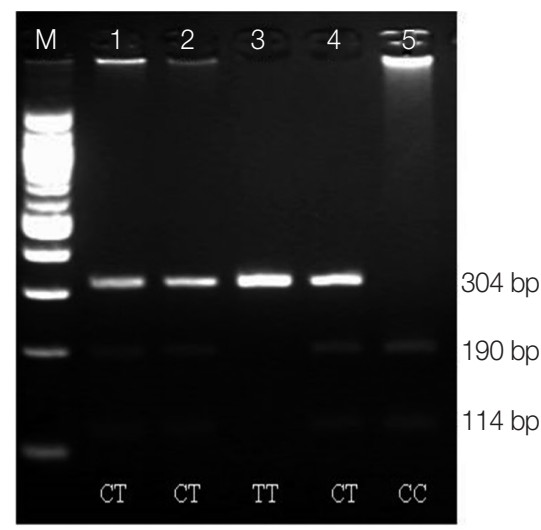

B

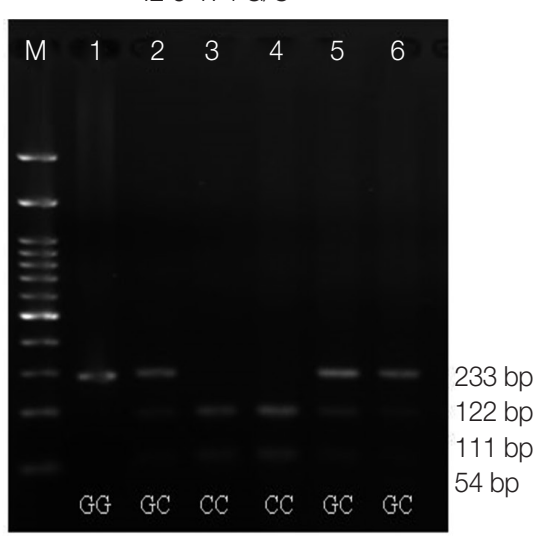

C IFN- $\gamma+874 \mathrm{~T} / \mathrm{A}$

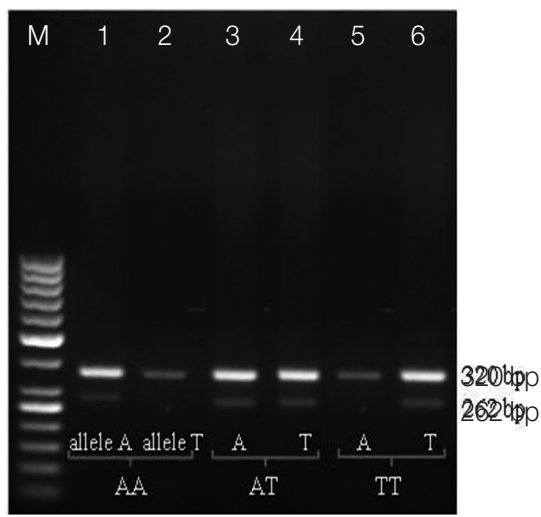

D

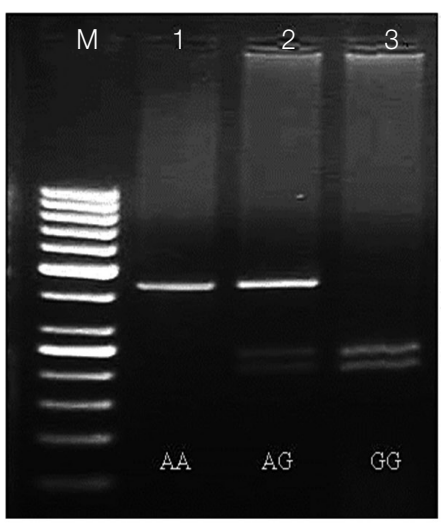

E TNF- $\alpha-308 G / A$

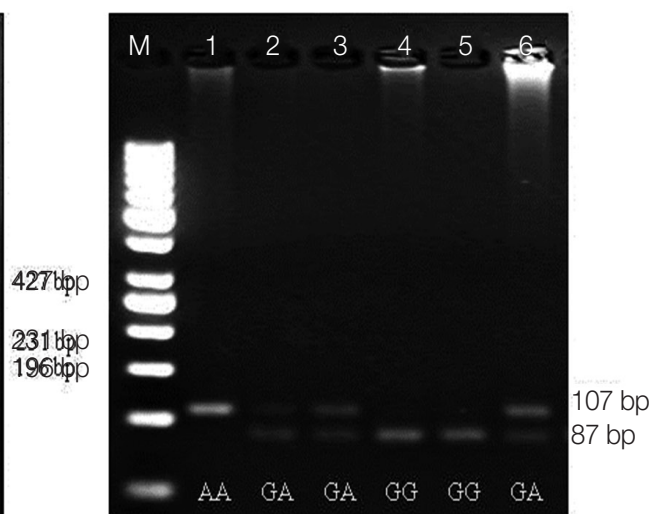

Fig. 1. Representative amplicons and digestion fragments corresponding to gene polymorphisms. (A) 190 and 114 bp fragments specific for C allele, 304 bp fragment specific for T allele of IL-1b-511C/T polymorphism. (B) 233 bp and 54 bp bands specific for $\mathrm{G}$ allele, 122 bp, 111 bp, and 54 bp bands specific for $C$ allele of IL-6-174G/C polymorphism. C) 262 bp fragment specific for T and A alleles of IFN- $\gamma+874 T / A$ polymorphism. (D) 427 bp fragment specific for A allele, 231 and 196 bp fragments specific for $G$ allele of LT-a +252A/G polymorphism. (E) 107 bp fragment specific for A allele, 87 bp fragments specific for $\mathrm{G}$ allele of TNF-a -308G/A polymorphism. M, 50 bp DNA marker.

Drop ND-1000 and stored at $-20^{\circ} \mathrm{C}$. Single nucleotide polymorphisms (SNPs) for IL-1 $\beta$-511C/T, IL-6 -174G/C, LT- $\alpha$
$+252 \mathrm{~A} / \mathrm{G}$, and TNF- $\alpha$-308G/A polymorphisms were analyzed by restriction fragment length polymorphism (RFLP), as de- 
scribed [15-18]. Allele specific-PCR (AS-PCR) was used to analyze for IFN- $\gamma+874$ T/A polymorphism [19]. Table 1 lists the PCR thermal cycling conditions and oligonucleotide primers specific for 5 cytokine gene polymorphisms, IL-1 $\beta-511 \mathrm{C} / \mathrm{T}$, IL-6 -174G/C, IFN- $\gamma+874$ T/A, LT- $\alpha+252 \mathrm{~A} / \mathrm{G}$, and TNF- $\alpha$ $-308 \mathrm{G} / \mathrm{A}$. Amplified products were evaluated by $1.5 \%(\mathrm{IFN}-\gamma)$, $3.0 \%$ (IL-6, LT- $\alpha$ and TNF- $\alpha$ ), and $8.0 \%$ (IL-1 $\beta$ ) agarose gel electrophoresis containing ethidium bromide $(0.5 \mu \mathrm{g} / \mathrm{ml})$. After electrophoresis, the ethidium-stained fragments were visualized and documented (Multi Doc-It ${ }^{\mathrm{TM}}$ Digital Imaging System, UVP, Upland, California, USA) (Fig. 1).

\section{Statistical analysis}

The Kolmogorov-Smirnov test was used to test whether the data were distributed normally. Statistical analysis was performed with non-parametric tests (Kruskal-Wallis and MannWhitney), with assistance of the IBM SPSS Statistics for Windows package, version 19 (SPSS, Chicago, Illinois, USA). Hardy-Weinberg equilibrium (HWE) analyses (chi-square tests, $\chi^{2}$ ) were used to compare the expected and observed genotype frequencies in the non-CCA groups of study participants. Allele frequency differences between cases (CCA) and controls (non-CCA) were analyzed, and the odd ratios (OR) and 95\% confidence intervals $(95 \% \mathrm{CI})$ were calculated.

\section{RESULTS}

\section{Demographic and clinicopathological characteristics}

Table 2 summarizes the clinicopathological characteristics of participants in this study who had been diagnosed with CCA as well as the liver fluke infected cases without CCA. The APF- group included 109 females and 91 males, mean age of 46.8 years (range, 20-60 years) and the APF+ group included 108 females and 92 males, mean age of 47.4 years (range, 2560 years). The CCA group was composed of 72 men and 38 women, and the mean age was 55.9 years (range, $40-76$ years).

\section{Cytokine production in Ov-ES stimulated PBMCs}

Fig. 2 summarizes the net production of all cytokines quantified following $\mathrm{O} v$-ES stimulation of PBMC from the APF-, $\mathrm{APF}+$, and CCA cases. Levels of IL-1 $\beta$, IFN- $\gamma$, and LT- $\alpha$ were significantly higher in CCA patients compared with the APFand $\mathrm{APF}+$ groups. TNF- $\alpha$ production was significantly higher in CCA and APF+ groups compared with APF-. Notably, IL-6 was significantly higher in $\mathrm{APF}+$ cases compared with the CCA
Table 2. Clinicopathological characteristics of cholangiocarcinoma (CCA) patients and non-CCA controls

\begin{tabular}{lccc}
\hline Demographics & APF- $(n=200)$ & APF $^{+}(n=200)$ & CCA $(n=110)$ \\
\hline Sex & & & \\
$\quad$ Male (\%) & $91(45.5)$ & $92(46.0)$ & $72(65.5)$ \\
$\quad$ Female (\%) & $109(54.5)$ & $108(54.0)$ & $38(34.5)$ \\
Age (years) & & & \\
$\quad$ Mean \pm SD & $46.8 \pm 7.7$ & $47.4 \pm 7.5$ & $55.9 \pm 10.3$ \\
Range & $20-60$ & $22-60$ & $40-76$ \\
Age (in year; \%) & & & \\
$\leq 30$ & $6(3.0)$ & $5(2.5)$ & $1(0.9)$ \\
$31-40$ & $34(17.0)$ & $33(16.5)$ & $5(4.6)$ \\
$41-50$ & $86(43.0)$ & $83(41.5)$ & $20(18.2)$ \\
$\geq 51$ & $74(37.0)$ & $79(39.5)$ & $84(46.4)$ \\
\hline
\end{tabular}

and APF- groups. Differences were not apparent among the groups for 6 other cytokines, IL-2, IL-4, IL-5, IL-8, IL-10, and IL-12p70.

\section{Gene polymorphism and cytokine production}

Overall comparison regardless of CCA or non-CCA groups did not reveal significant differences in levels of cytokines among gene polymorphisms except for LT- $\alpha$, where net cytokine production in LT- $\alpha+252 \mathrm{~A} / \mathrm{G}$ heterozygous (AG) and homozygous (GG) variants were significantly elevated compared to the wild type (Fig. 3). However, when subdividing into CCA and non-CCA groups (APF- and APF+), LT- $\alpha+254 \mathrm{~A} / \mathrm{G}$ heterozygous (AG), and homozygous (GG) variants showed significantly higher levels of cytokines than the wild type in CCA group; 2.35-fold and 2.24-fold, respectively. Similarly, TNF- $\alpha$ 308G/A heterozygous (GA) and homozygous (AA) variants showed significantly higher levels of cytokines than the wild type in CCA group with fold changes of 12.49 and 4.76, respectively. Interestingly, IFN- $\gamma+874 \mathrm{~T}>\mathrm{A}$ heterozygous (TA) and homozygous (AA) was significantly lower (0.56- and 0.2fold, respectively) than the wild type in the CCA group.

\section{Distribution of cytokine gene polymorphisms among patient groups}

Table 4 presents the genotypes and allele distribution of IL$1 \beta-511 \mathrm{C} / \mathrm{T}$, IL-6 - $174 \mathrm{G} / \mathrm{C}$, IFN- $\gamma+874 \mathrm{~T} / \mathrm{A}, \mathrm{LT}-\alpha+254 \mathrm{~A} / \mathrm{G}$, and TNF- $\alpha-308 \mathrm{G} / \mathrm{A}$ polymorphisms in the participants, those with $\mathrm{APF}-\mathrm{APF}+$, and CCA. Comparison among groups did not reveal significant differences in the genotype or allele frequencies for IL-1 $\beta-511 \mathrm{C} / \mathrm{T}$, LT- $\alpha+252 \mathrm{~A} / \mathrm{G}$, and TNF- $\alpha-308 \mathrm{G} / \mathrm{A}$. However, comparing the IL6 $-174 \mathrm{G} / \mathrm{C}$ genotypes between the $\mathrm{CCA}$ and non-CCA (APF- or APF+) groups revealed that the presence of heterozygous (GC) genotype associated with CCA 

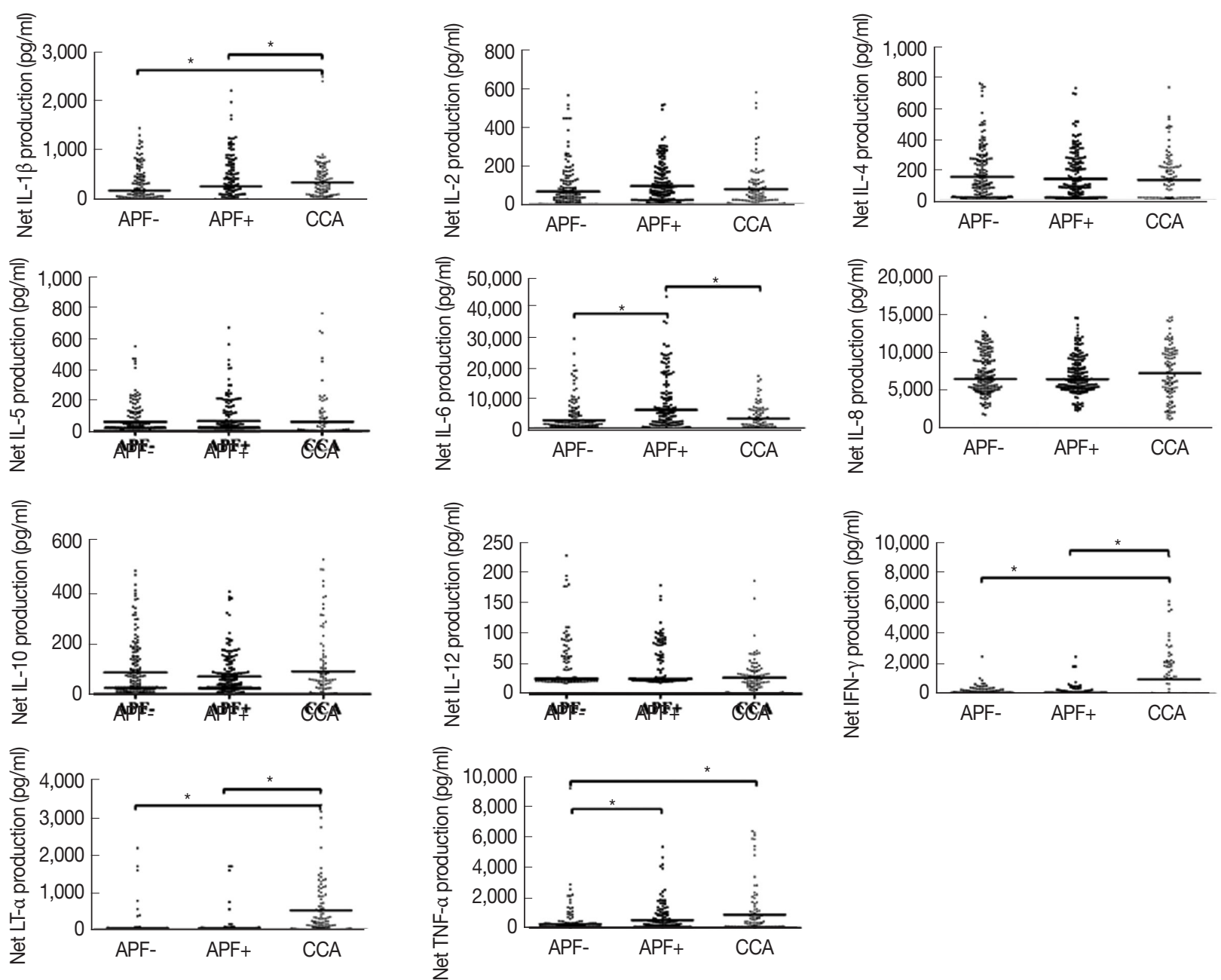

Fig. 2. Net cytokine profile of OvES stimulated PBMCs in APF-, APF+, and CCA patients. The Wilcoxon Matched-paired Signed rank test was used to assess the statistical significance; ${ }^{*} P<0.05$.

$(\mathrm{OR}=2.35$ and 2.95 for APF- vs CCA and APF+ vs CCA, respectively). Moreover, a stronger association with CCA was found with the homozygous variant (CC) $(\mathrm{OR}=5.50$ and 7.52 for APF- vs CCA and APF+ vs CCA, respectively). In addition, $\mathrm{C}$ alleles at this locus also associated with CCA $(\mathrm{OR}=3.36)$ when the CCA and $\mathrm{APF}+$ groups were compared. Comparing between $\mathrm{APF}+$ and $\mathrm{APF}$ - groups, IFN- $\gamma+874 \mathrm{~T}>\mathrm{A}$ heterozygous (TA) and homozygous (AA) variants associated with biliary periductal fibrosis ( $\mathrm{OR}=3.10$ and 2.74, respectively). The IFN$\gamma+874 \mathrm{~T}>\mathrm{A}$ homozygous variant (AA) genotype was also associated with CCA $(\mathrm{OR}=2.20)$ as compared between APF- and CCA groups, whereas the A allele associated with both fibrosis $(\mathrm{OR}=1.60, \mathrm{APF}-\mathrm{vs} \mathrm{APF}+)$ and $\mathrm{CCA}(\mathrm{OR}=1.71, \mathrm{APF}-\mathrm{vs} \mathrm{CCA})$. In the case of TNF- $\alpha-308 \mathrm{G} / \mathrm{A}$ polymorphism, only the TNF- $\alpha$ -308 A allele appeared to be associated with CCA (OR=1.61 and $\mathrm{OR}=1.5$ in APF- vs CCA and in APF+ vs CCA, respectively).
Table 3. Cytokine levels as established in PBMCs pulsed with excretory secretory profcuts of the liver fluke Opisthorchis viverrini and the net cytokine production and genotype polymorphisms

\begin{tabular}{lccc}
\hline & \multicolumn{3}{c}{ Cytokine levels, pg/ml } \\
\cline { 2 - 4 } & Wild type & Heterozygous & Variant \\
\hline IL-1 $\beta$ & $268.4 \pm 47.7$ & $315.4 \pm 35.9$ & $303.1 \pm 41.1$ \\
IL-6 & $3,973.1 \pm 763.2$ & $4,221.6 \pm 434.8$ & $4,323.7 \pm 440.5$ \\
IFN- $\gamma$ & $514.9 \pm 140.3$ & $397.5 \pm 117.4$ & $239.9 \pm 65.2$ \\
LT- $a$ & $67.3 \pm 17.9$ & $187.4 \pm 36.0^{*}$ & $153.7 \pm 36.7^{*}$ \\
TNF- $\alpha$ & $362.6 \pm 68.8$ & $498.8 \pm 127.1$ & $466.7 \pm 67.9$ \\
\hline
\end{tabular}

Cytokine levels presented as mean $\pm \mathrm{SD} ;{ }^{\star} P<0.05 ; \mathrm{n}=510$.

\section{DISCUSSION}

Infection with the liver fluke O. viverrini frequently leads to chronic inflammation of the bile ducts and results in unremitting tissue damage that, in turn, can cause hepatobiliary dis- 
APF-
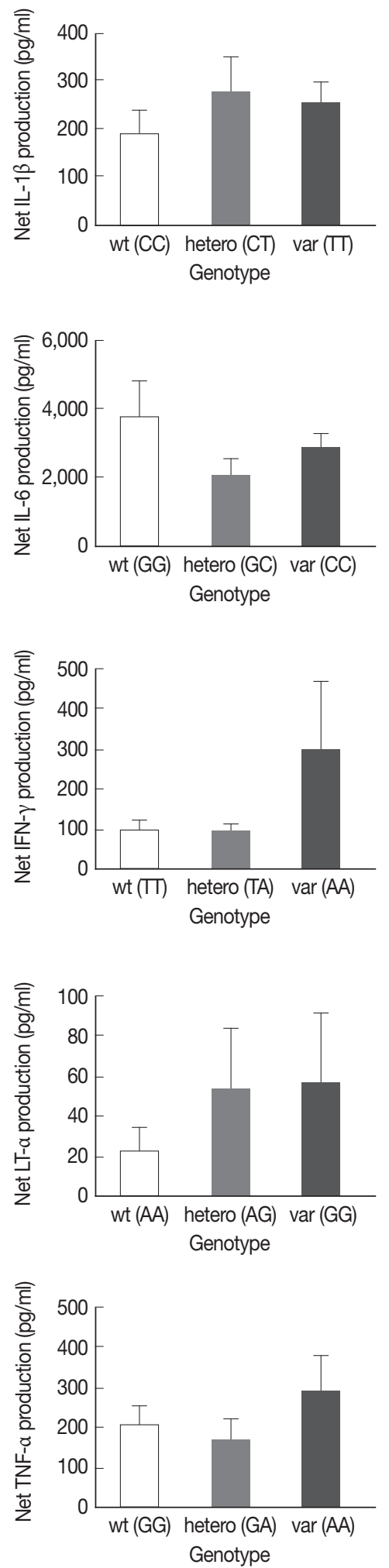

APF+
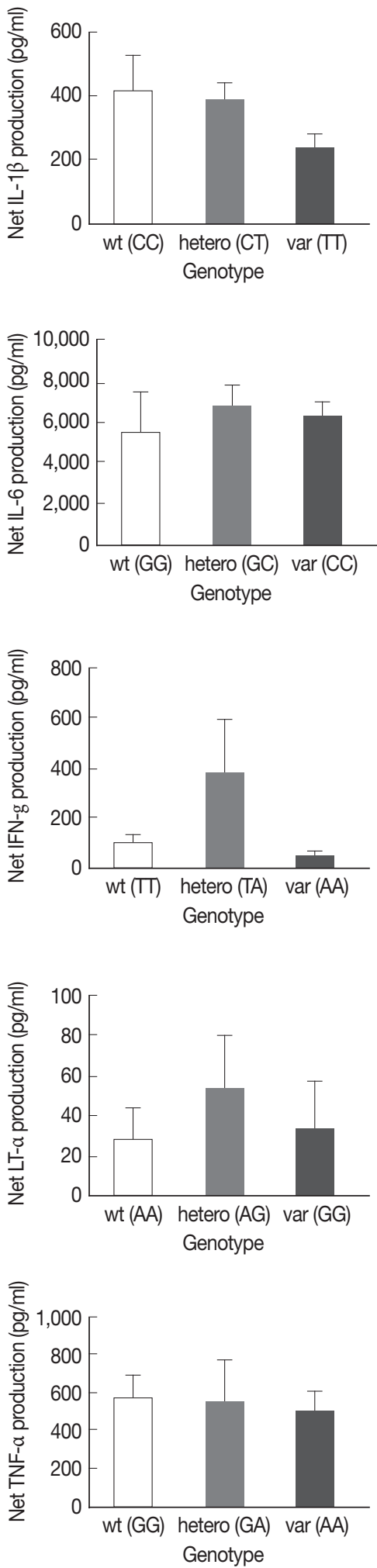

CCA
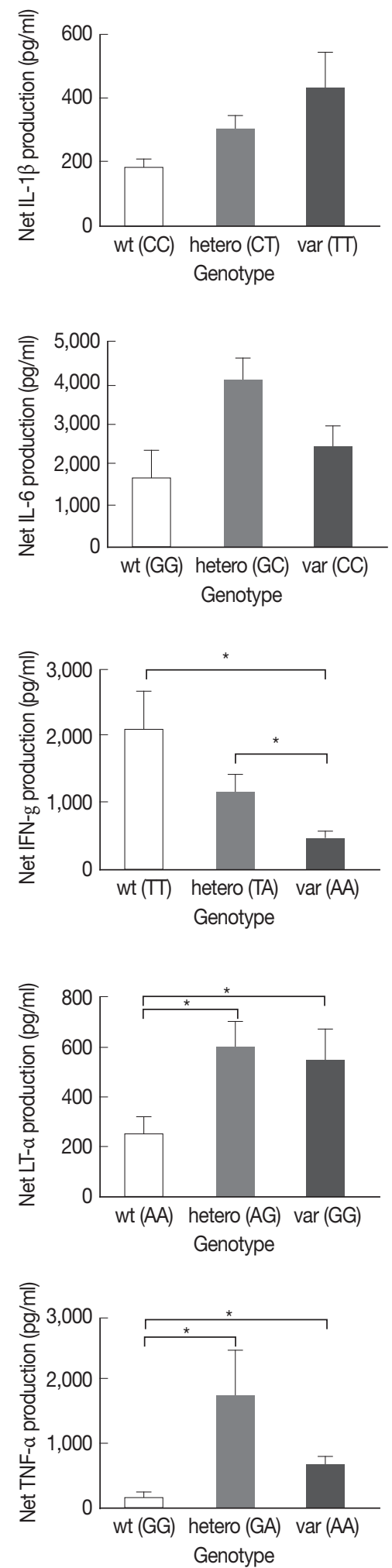

Fig. 3. The net production of $I L-1 \beta, \| L-6, I F N-\gamma, L T-a$, and TNF- $a$, and their genotype polymorphisms in $A P F-(n=200), A P F+(n=200)$, and CCA patients $(n=110)$. wt, wild type; hetero, heterozygous; var, variant. 
Table 4. Comparison of genotypes and allele frequencies for IL-1 $\beta, \| L-6, I F N-\gamma, L T-a$, and TNF- $\alpha$ in CCA cases and the participants in the APF+ and the APF- groups

\begin{tabular}{|c|c|c|c|c|c|c|}
\hline \multirow{2}{*}{$\begin{array}{l}\text { Cytokine/ } \\
\text { Genotype/Allele }\end{array}$} & \multirow{2}{*}{ APF-N(\%) } & \multirow{2}{*}{$\mathrm{APF}+\mathrm{N}(\%)$} & \multirow{2}{*}{ CCA N(\%) } & \multirow{2}{*}{$\frac{\text { APF- vs. APF+ }}{\text { OR }(95 \% \mathrm{Cl})}$} & \multirow{2}{*}{$\begin{array}{c}\text { APF- vs. CCA } \\
\text { OR }(95 \% \mathrm{Cl})\end{array}$} & \multirow{2}{*}{$\frac{\text { APF + vs. CCA }}{\text { OR }(95 \% \text { Cl) }}$} \\
\hline & & & & & & \\
\hline \multicolumn{7}{|l|}{$\mathrm{IL}-1 \beta(-511 \mathrm{C} / \mathrm{T})$} \\
\hline CC & 38 (19.0) & 36 (18.0) & $18(16.1)$ & 1 & 1 & 1 \\
\hline $\mathrm{CT}$ & $100(50.0)$ & 93 (46.5) & 48 (43.6) & $0.98(0.50-1.74)$ & $1.01(0.50-2.09)$ & $1.03(0.50-2.14)$ \\
\hline$\pi$ & $62(31.0)$ & $71(35.5)$ & $44(40.0)$ & $1.20(0.65-2.22)$ & $1.49(0.72-3.16)$ & $1.23(0.59-2.61)$ \\
\hline $\mathrm{C}$ & $176(44.0)$ & $165(41.2)$ & $84(38.2)$ & 1 & 1 & 1 \\
\hline $\mathrm{T}$ & $244(56.0)$ & $235(58.8)$ & $136(61.8)$ & $1.02(0.77-1.36)$ & $1.16(0.82-1.65)$ & $1.13(0.80-1.61)$ \\
\hline \multicolumn{7}{|l|}{ IL-6 (-174G/C) } \\
\hline GG & $112(56.0)$ & $124(62.0)$ & $32(29.1)$ & 1 & 1 & 1 \\
\hline $\mathrm{GC}$ & 67 (33.5) & 59 (29.5) & 45 (40.9) & $0.79(0.50-1.25)$ & $2.35(1.31-4.21)^{\star}$ & $2.95(1.64-5.31)^{\star}$ \\
\hline $\mathrm{CC}$ & $21(10.5)$ & $17(8.5)$ & $33(30.0)$ & $0.73(0.34-1.53)$ & $5.50(2.65-11.41)^{\star}$ & $7.52(3.52-16.19)^{*}$ \\
\hline G & 291 (72.7) & 307 (76.7) & $109(49.5)$ & 1 & 1 & 1 \\
\hline C & 109 (27.3) & 93 (23.3) & $111(50.5)$ & $0.81(0.58-1.13)$ & $0.26(0.12-0.52)$ & $3.36(2.32-4.85)^{\star}$ \\
\hline \multicolumn{7}{|l|}{ IFN- $\gamma(+874 \mathrm{~T} / \mathrm{A})$} \\
\hline$\pi$ & $61(30.5)$ & $26(13.0)$ & 23 (20.9) & 1 & 1 & 1 \\
\hline TA & $74(37.0)$ & 98 (49.0) & $33(30.0)$ & $3.10(1.73-5.61)^{\star}$ & $1.18(0.60-2.34)$ & $0.38(0.18-0.80)$ \\
\hline $\mathrm{AA}$ & 65 (32.5) & 76 (38.0) & 54 (49.1) & $2.74(1.50-5.04)^{\star}$ & $2.20(1.13-4.20)^{\star}$ & $0.80(0.39-1.64)$ \\
\hline $\mathrm{T}$ & 196 (49.0) & $150(37.5)$ & 79 (35.9) & 1 & 1 & 1 \\
\hline A & 204 (51.0) & $250(62.5)$ & $141(64.1)$ & $1.60(1.19-2.14)^{\star}$ & $1.71(1.20-2.44)^{\star}$ & $1.07(0.75-1.5)$ \\
\hline \multicolumn{7}{|l|}{ LT-a (+252A/G) } \\
\hline $\mathrm{AA}$ & $60(30.0)$ & 47 (23.5) & 24 (21.8) & 1 & 1 & 1 \\
\hline$A G$ & $74(37.0)$ & 93 (46.5) & $54(49.1)$ & $1.60(0.95-2.65)$ & $1.84(0.97-3.45)$ & $1.17(0.60-2.16)$ \\
\hline GG & $66(33.0)$ & $60(30.0)$ & $32(29.1)$ & $1.16(0.66-2.01)$ & $1.21(0.61-2.40)$ & $1.04(0.51-2.11)$ \\
\hline A & $194(48.5)$ & $187(46.8)$ & $102(46.4)$ & 1 & 1 & 1 \\
\hline G & $206(51.5)$ & $213(53.2)$ & 118 (53.6) & $1.07(0.80-1.43)$ & $1.08(0.77-1.53)$ & $1.01(0.72-1.43)$ \\
\hline \multicolumn{7}{|l|}{ TNF-a $(-308 G / A)$} \\
\hline GG & $34(17.0)$ & 35 (17.5) & $17(15.5)$ & 1 & 1 & 1 \\
\hline $\mathrm{GA}$ & $54(27.0)$ & $46(23.0)$ & $13(11.8)$ & $0.82(0.42-1.60)$ & $0.48(0.18-1.20)$ & $0.58(0.22-1.47)$ \\
\hline$A A$ & $112(56.0)$ & $119(59.5)$ & $80(72.7)$ & $1.03(0.58-1.83)$ & $1.42(0.71-2.92)$ & $1.38(0.69-2.82)$ \\
\hline$G$ & $122(30.5)$ & $116(29.0)$ & 47 (21.4) & 1 & 1 & 1 \\
\hline$A$ & 278 (69.5) & $284(71.0)$ & $173(78.6)$ & $1.07(0.78-1.47)$ & $1.61(1.08-2.43)^{\star}$ & $1.50(1.00-2.26)^{\star}$ \\
\hline
\end{tabular}

*Significant at $P$-value $<0.05$.

eases including CCA. A subset of people with opisthorchiasis develop overt hepatobiliary disease, with about $25 \%$ of them displaying marked biliary tract fibrosis and as many as $0.5 \%$ of infected cases exhibiting incipient bile duct cancer upon ultrasound examination $[4,20,21]$. Why infection leads to different disease outcomes among the population in high liver fluke infection endemic sites remains to be established. Divergent immunological responses among infected persons may contribute to the outcome, in addition to other co-factors [22]. The pro-inflammatory cytokine IL-6 is significantly elevated in infected individuals with marked and chronic hepatobiliary fibrosis $[9,23]$ and cholangiocytes upregulate expression of IL- 6 and IL-8 in response to the $\mathrm{O} v$-ES components [24,25]. Moreover, the recent study revealed both pro-inflammatory and anti-inflammatory cytokines were increased in the $O$. viverrini-associated CCA compared to uninfected normal controls [10].

Here, we investigated pro-inflammatory and anti-inflamma- tory cytokine profiles among different groups of patients infected with $O$. viverrini: liver fluke infected individuals exhibiting biliary tract disease (advanced periductal fibrosis, $\mathrm{APF}+$ ) or without overt biliary periductal fibrosis (APF-), and liver fluke infection-associated CCA cases. We observed that pro-inflammatory cytokines, IL- $1 \beta$, IL- 6 , IFN- $\gamma$, LT- $\alpha$, and TNF- $\alpha$, though no other cytokines were increased significantly during CCA in comparison with participants infected with $O$. viverrini but without CCA, i.e., the APF- and APF+ groups. These findings indicated that marked inflammatory responses during opisthorchiasis increased the risk for cholangiocarcinogenesis.

Gene polymorphisms are known to affect cytokine levels $[8,26,27]$. Single nucleotide polymorphism (SNP) can affect gene function in many ways [28]. If it occurs at the non-coding region, it can affect the binding of transcriptional factors, interfere RNA splicing, and increase or decrease messenger RNA degradation. SNP in coding region can result in 2 consequenc- 
es; synonymous and nonsynonymous SNPs. Synonymous SNPs do not affect the protein sequence, but can still affect the protein conformation such as the silent polymorphism in the MDR1 gene changes substrate specificity [29]. Nonsynonymous SNPs change the amino acid sequence of protein and subsequent protein function as shown in cystic fibrosis patients [30]. For SNPs in our liver fluke associated CCA, we report here that certain cytokine gene polymorphisms affect proinflammatory cytokine production. However, detail molecular mechanisms need to be further investigated.

Accordingly, we hypothesized that cytokine gene polymorphisms may underline variations in inflammatory cytokine response in response to liver fluke infection and downstream biliary tract inflammation and cholangiocarcinogenesis. The gene polymorphisms IL-1 $\beta$-511C/T, IL6 -174G/C, LT- $\alpha+252 \mathrm{~A} /$ $\mathrm{G}$, and TNF- $\alpha-308 \mathrm{G} / \mathrm{A}$ generated the high-producer phenotypes, whereas IFN- $\gamma+874 \mathrm{~T} / \mathrm{A}$ exhibited a low-production phenotype. In agreement with these reports, both LT- $\alpha+252 \mathrm{~A} / \mathrm{G}$ and TNF- $\alpha-308 \mathrm{G} / \mathrm{A}$ heterozygous and homozygous variants showed significant higher levels of cytokine than the wild type. Similarly, both IL-1 $\beta$-511C/T and IL-6 - $174 \mathrm{G} / \mathrm{C}$ variants produced more cytokines than the wild type, even though the difference was not statistically significant. By contrast, the variants of IFN- $\gamma+874 \mathrm{~T} / \mathrm{A}$ produced less cytokines than the wild type. We note, however, that these cytokine production profiles were observed only in the CCA cases, and not among the non-CCA groups. The difference of cytokine production from the same gene polymorphism between non-CCA and CCA patients might indicate divergence in cytokine regulation/networking among the groups in this study.

Subsequently we investigated whether gene polymorphisms of these pro-inflammatory cytokine were related to the development of fibrosis or CCA. An IL-6 -174G > C polymorphism represents a potential modulator of risk for malignancy with ethnicity and tumor site dependent trends [31]. The present findings revealed that both heterozygous (GC) and homozygous (CC) variant of IL6 -174G>C polymorphisms were associated with cholangiocarcinogenesis in this Thai population. Given that the heterozygous (GC) and homozygous (CC) variants of IL6 -174G > C are high producer phenotypes, these gene polymorphisms might explain elevated levels of IL- 6 during in CCA, as reported earlier. The A allele of the TNF- $\alpha$ $-308 \mathrm{G} / \mathrm{A}$ polymorphism appears to associate with the disease progression of hepatitis B viral infection and hepatocellular carcinoma [32]. In likely fashion, the present findings revealed that the A allele of TNF- $\alpha-308 \mathrm{G} / \mathrm{A}$ polymorphism associated with CCA.

Interferon gamma plays key roles in the immune responses and in antitumor mechanisms [33,34]. IFN- $\gamma+874 \mathrm{~T}>$ A polymorphisms are associated with viral and bacterial infections, which result in chronic inflammation and carcinogenic [3537]. Here, IFN- $\gamma+874 \mathrm{~T}>\mathrm{A}$ heterozygous (TA) and homozygous (AA) variants associated with fibrosis, and the IFN$\gamma+874 \mathrm{~T}>\mathrm{A}$ homozygous (AA) variant was also associated with CCA. As noted, these variants produce less IFN- $\gamma$ than the TT (wild type) genotype. Together these findings indicate that in a subset of liver fluke infected individuals carrying these alleles, the phenotype of which is reduced levels of IFN- $\gamma$, are at elevated risk for biliary periductal fibrosis and CCA. Mechanisms underlying the association are not known. However, for antitumor activity, IFN- $\gamma$ plays a central role in the recognition and elimination of transformed cells through its cytostatic, proapoptotic, and immune-provoking effects [33].

To summarize, cytokine and gene polymorphism profiles of Thais infected with the liver fluke $O$. viverrini were characterized. To our knowledge, this is the first demonstration that persons infected with $O$. viverrini inherited with certain gene polymorphisms that result in aberrant cytokine production are susceptible to develop fibrosis and CCA. Pro-inflammatory cytokines were increased in persons with cholangiocarcinoma (CCA). Different cytokine gene polymorphisms were found among patients with or without fibrosis and CCA. Interestingly, a subset of patients with the IL6 -174G > C polymorphisms, the IFN $-\gamma+874 \mathrm{~T}>\mathrm{A}$ heterozygous (TA) and homozygous (AA) variants, and the A allele of TNF- $\alpha-308 \mathrm{G} / \mathrm{A}$ associated with $\mathrm{CCA}$. Further studies on these gene polymorphisms and in relation to other cytokines are warranted since they may identify genotypes and phenotypes predictive of risk for CCA.

\section{ACKNOWLEDGMENTS}

This work was supported by the Higher Education Research Promotion and National Research University Project of Thailand, Office of the Higher Education Commission, through the Health Cluster (SHeP-GMS), Khon Kaen University, the Thailand Research Fund (TRF) grant no. RTA 5680006, the National Institute of Allergy and Infectious Diseases (NIAID), NIH, award no. P50AI098639, and National Cancer Institute (NCI), National Institutes of Health (NIH) award no. CA1 64719. B. Sripa is a TRF Senior Research Scholar. The content is 
solely the responsibility of the authors and does not necessarily represent the official views of the TRF, NIAID, NCI, or the $\mathrm{NIH}$ or the funders.

\section{CONFLICT OF INTEREST}

We have no conflict of interest related to this study.

\section{REFERENCES}

1. Sripa B, Brindley PJ, Mulvenna J, Laha T, Smout MJ, Mairiang E, Bethony JM, Loukas A. The tumorigenic liver fluke Opisthorchis viverrini-multiple pathways to cancer. Trends Parasitol 2012; 28: 395-407.

2. Sripa B, Kaewkes S, Sithithaworn P, Mairiang E, Laha T, Smout M, Pairojkul C, Bhudhisawasdi V, Tesana S, Thinkamrop B, Bethony JM, Loukas A, Brindley PJ. Liver fluke induces cholangiocarcinoma. PLoS Med 2007; 4: e201.

3. Mairiang E, Chaiyakum J, Chamadol N, Laopaiboon V, Srinakarin J, Kunpitaya J, Sriamporn S, Suwanrungruang K, Vatanasapt V. Ultrasound screening for Opisthorchis viverrini-associated cholangiocarcinomas: experience in an endemic area. Asian Pac J Cancer Prev 2006; 7: 431-433.

4. Mairiang E, Laha T, Bethony JM, Thinkhamrop B, Kaewkes S, Sithithaworn P, Tesana S, Loukas A, Brindley PJ, Sripa B. Ultrasonography assessment of hepatobiliary abnormalities in 3359 subjects with Opisthorchis viverrini infection in endemic areas of Thailand. Parasitol Int 2012; 61: 208-211.

5. Bradley JR. TNF-mediated inflammatory disease. J Pathol 2008; 214: 149-160.

6. Coussens LM, Werb Z. Inflammation and cancer. Nature 2002; 420: 860-867.

7. Ninlawan K, O'Hara SP, Splinter PL, Yongvanit P, Kaewkes S, Surapaitoon A, LaRusso NF, Sripa B. Opisthorchis viverrini excretory/secretory products induce toll-like receptor 4 upregulation and production of interleukin 6 and 8 in cholangiocyte. Parasitol Int 2010; 59: 616-621.

8. Bidwell J, Keen L, Gallagher G, Kimberly R, Huizinga T, McDermott MF, Oksenberg J, McNicholl J, Pociot F, Hardt C, D'Alfonso S. Cytokine gene polymorphism in human disease: on-line databases. Genes Immun 1999; 1: 3-19.

9. Sripa B, Mairiang E, Thinkhamrop B, Laha T, Kaewkes S, Sithithaworn P, Tessana S, Loukas A, Brindley PJ, Bethony JM. Advanced periductal fibrosis from infection with the carcinogenic human liver fluke Opisthorchis viverrini correlates with elevated levels of interleukin-6. Hepatology 2009; 50: 1273-1281.

10. Surapaitoon A, Suttiprapa S, Khuntikeo N, Pairojkul C, Sripa B. Cytokine profiles in Opisthorchis viverrini stimulated peripheral blood mononuclear cells from cholangiocarcinoma patients. Parasitol Int 2016; 66: 889-892.

11. Sripa B, Kaewkes S. Localisation of parasite antigens and inflam- matory responses in experimental opisthorchiasis. Int J Parasitol 2000; 30: 735-740.

12. Sripa B, Kaewkes S. Relationship between parasite-specific antibody responses and intensity of Opisthorchis viverrini infection in hamsters. Parasite Immunol 2000; 22: 139-145.

13. Aida Y, Pabst MJ. Removal of endotoxin from protein solutions by phase separation using Triton X-114. J Immunol Methods 1990; 132: 191-195.

14. Hurley JC. Endotoxemia: methods of detection and clinical correlates. Clin Microbiol Rev 1995; 8: 268-292.

15. Duarte I, Santos A, Sousa H, Catarino R, Pinto D, Matos A, Pereira D, Moutinho J, Canedo P, Machado JC, Medeiros R. G308A TNF-alpha polymorphism is associated with an increased risk of invasive cervical cancer. Biochem Biophys Res Commun 2005; 334: 588-592.

16. Gatti LL, Burbano RR, Zambaldi-Tunes M, de-Lábio RW, de Assumpção PP, de Arruda Cardoso-Smith M, Marques-Payão SL. Interleukin-6 polymorphisms, Helicobacter pylori infection in adult Brazilian patients with chronic gastritis and gastric adenocarcinoma. Arch Med Res 2007; 38: 551-555.

17. He B, Pan Y, Xu Y, Nie Z, Chen L, Gu L, Wang S. Increased risk for gastric cancer in carriers of the lymphotoxin-alpha+252G variant infected by Helicobacter pylori. Genet Test Mol Biomarkers 2012; 16: 9-14.

18. Rad R, Prinz C, Neu B, Neuhofer M, Zeitner M, Voland P, Becker I, Schepp W, Gerhard M. Synergistic effect of Helicobacter pylori virulence factors and interleukin-1 polymorphisms for the development of severe histological changes in the gastric mucosa. J Infect Dis 2003; 188: 272-281.

19. Pravica V, Perrey C, Stevens A, Lee JH, Hutchinson IV. A single nucleotide polymorphism in the first intron of the human IFNgamma gene: absolute correlation with a polymorphic CA microsatellite marker of high IFN-gamma production. Hum Immunol 2000; 61: 863-866.

20. Khuntikeo N, Loilome W, Thinkhamrop B, Chamadol N, Yongvanit P. A comprehensive public health conceptual framework and strategy to effectively combat cholangiocarcinoma in Thailand. PLoS Negl Trop Dis 2016; 10: e0004293.

21. Sungkasubun P, Siripongsakun S, Akkarachinorate K, Vidhyarkorn S, Worakitsitisatorn A, Sricharunrat T, Singharuksa S, Chanwat R, Bunchaliew C, Charoenphattharaphesat S, Molek R, Yimyaem M, Sornsamdang G, Soonklang K, Wittayasak K, Auewarakul CU, Mahidol C. Ultrasound screening for cholangiocarcinoma could detect premalignant lesions and early-stage diseases with survival benefits: a population-based prospective study of 4,225 subjects in an endemic area. BMC Cancer 2016; 16: 346 .

22. Brindley PJ, da Costa JM, Sripa B. Why does infection with some helminths cause cancer? Trends Cancer 2015; 1: 174-182.

23. Sripa B, Thinkhamrop B, Mairiang E, Laha T, Kaewkes S, Sithithaworn P, Periago MV, Bhudhisawasdi V, Yonglitthipagon P, Mulvenna J, Brindley PJ, Loukas A, Bethony JM. Elevated plasma IL-6 associates with increased risk of advanced fibrosis and chol- 
angiocarcinoma in individuals infected by Opisthorchis viverrini. PLoS Negl Trop Dis 2012; 6: e1654.

24. Chaiyadet S, Smout M, Johnson M, Whitchurch C, Turnbull L, Kaewkes S, Sotillo J, Loukas A, Sripa B. Excretory/secretory products of the carcinogenic liver fluke are endocytosed by human cholangiocytes and drive cell proliferation and IL6 production. Int J Parasitol 2015; 45: 773-781.

25 Smout MJ, Sotillo J, Laha T, Papatpremsiri A, Rinaldi G, Pimenta RN, Chan LY, Johnson MS, Turnbull L, Whitchurch CB, Giacomin PR, Moran CS, Golledge J, Daly N, Sripa B, Mulvenna JP, Brindley PJ, Loukas A. Carcinogenic parasite secretes growth factor that accelerates wound healing and potentially promotes neoplasia. PLoS Pathog 2015; 11: e1005209.

26. Cantor MJ, Nickerson P, Bernstein CN. The role of cytokine gene polymorphisms in determining disease susceptibility and phenotype in inflammatory bowel disease. Am J Gastroenterol 2005; 100: 1134-1142.

27. Dai CY, Chuang WL, Hsieh MY, Lee LP, Hou NJ, Chen SC, Lin ZY, Hsieh MY, Wang LY, Tsai JF, Chang WY, Yu ML. Polymorphism of interferon-gamma gene at position +874 and clinical characteristics of chronic hepatitis C. Transl Res 2006; 148: 128133.

28. Shastry BS. SNPs: impact on gene function and phenotype. Methods Mol Biol 2009; 578: 3-22.

29. Kimchi-Sarfaty C, Oh JM, Kim IW, Sauna ZE, Calcagno AM, Ambudkar SV, Gottesman MM. A "silent" polymorphism in the MDR1 gene changes substrate specificity. Science 2007; 315: 525-528.
30. Cordovado SK, Hendrix M, Greene CN, Mochal S, Earley MC, Farrell PM, Kharrazi M, Hannon WH, Mueller PW. CFTR mutation analysis and haplotype associations in CF patients. Mol Genet Metab 2012; 105: 249-254.

31. Joshi N, Kannan S, Kotian N, Bhat S, Kale M, Hake S. Interleukin $6-174 \mathrm{G}>\mathrm{C}$ polymorphism and cancer risk: meta-analysis reveals a site dependent differential influence in Ancestral North Indians. Hum Immunol 2014; 75: 901-908.

32. Wang B, Wang J, Zheng Y, Zhou S, Zheng J, Wang F, Ma X, Zeng Z. A study of TNF- $\alpha-238$ and -308 polymorphisms with different outcomes of persistent hepatitis B virus infection in China. Pathology 2010; 42: 674-680.

33. Kursunel MA, Esendagli G. The untold story of IFN-gamma in cancer biology. Cytokine Growth Factor Rev 2016; 31: 73-81.

34. Pestka S, Krause CD, Walter MR. Interferons, interferon-like cytokines, and their receptors. Immunol Rev 2004; 202: 8-32.

35. Cardoso CC, Pereira AC, Brito-de-Souza VN, Dias-Baptista IM, Maniero VC, Venturini J, Vilani-Moreno FR, de Souza FC, Ribeiro-Alves M, Sarno EN, Pacheco AG, Moraes MO. IFNG +874 $\mathrm{T}>\mathrm{A}$ single nucleotide polymorphism is associated with leprosy among Brazilians. Hum Genet 2010; 128: 481-490.

36. Sun Y, Lu Y, Pen Q, Li T, Xie L, Deng Y, Qin A. Interferon gamma +874 T/A polymorphism increases the risk of cervical cancer: evidence from a meta-analysis. Tumour Biol 2015; 36: 4555-4564.

37. von Linsingen R, Bompeixe EP, Maestri CA, Carvalho NS, da Graca Bicalho M. IFNG (+874 T/A) polymorphism and cervical intraepithelial neoplasia in Brazilian women. J Interferon Cytokine Res 2009; 29: 285-288. 